disease. IL-21 was expressed at a high level in $\mathrm{CD}^{+}{ }^{+}$lymphocytes isolated from the lamina propria of untreated patients, indicating that these cells are responsible for this production of this cytokine.

The authors conclude that, in patients with active celiac disease, overproduction of IL-21 increases expression of IFN- $\gamma$ and T-bet, and thus is an important contributor to the mucosal $\mathrm{T}_{\mathrm{H}} 1$ proinflammatory response.

Original article Fina D et al. (2008) Interleukin 21 contributes to the mucosal $\mathrm{T}$ helper cell type 1 response in coeliac disease. Gut 57: 887-892

\section{Moderate wine drinking might decrease prevalence of nonalcoholic fatty liver disease}

Evidence of the cardioprotective effect of moderate wine consumption has led some clinicians to recommend such consumption to patients at risk of coronary heart disease. However, as these patients are also at risk of nonalcoholic fatty liver disease (NAFLD), is the recommendation safe? To find out, Dunn et al. tested the hypothesis that moderate wine drinking is associated with a reduced prevalence of NAFLD.

The cross-sectional, population-based study included participants of the Third National Health and Nutrition Examination Survey who were nondrinkers $(n=7,211)$ or who drank wine preferentially with a total alcohol consumption of up to $10 \mathrm{~g}$ per day $(n=945)$. After exclusion of individuals with other causes of serum alanine aminotranferase (ALT) elevation, wellknown ALT cutoff values were used to designate suspected NAFLD: ALT $>43 \mathrm{U} / \mathrm{l}$ or $>30 \mathrm{U} / \mathrm{I}$ (for men) and ALT >19 U/I (for women).

Moderate wine drinking was independently associated with a reduced prevalence of suspected NAFLD in a multivariate analysis that adjusted for social, demographic and lifestyle variables (adjusted odds ratios 0.15 and 0.51 for the higher and lower men's ALT cutoff values, respectively). Although moderate wine drinkers had fewer metabolic abnormalities than nondrinkers, analysis revealed that the protective effect of moderate wine drinking was independent of these variables.

The findings suggest that it is safe to drink up to one small glass of wine per day for cardioprotection and that this consumption might also protect against NAFLD. However, the researchers call for prospective studies to evaluate the full effect of moderate wine drinking on the liver.

Original article Dunn W et al. (2008) Modest wine drinking and decreased prevalence of suspected nonalcoholic fatty liver disease. Hepatology 47: 1947-1954

\section{Increased prevalence of HBV infection in patients with type 2 diabetes}

Immunosuppression is associated with HBV infection. Patients with type 2 diabetes mellitus (T2DM), who are known to be vulnerable to immune dysregulation, have an increased incidence of hepatocellular carcinoma. Although there are no previous reports of occult HBV infection in patients with T2DM, HBV is a possible cause of this increased incidence. Researchers in Turkey, therefore, investigated the prevalence of $\mathrm{HBV}$ infection in patients with T2DM. The study showed that occult HBV infection was significantly more prevalent in patients with T2DM than in nondiabetic controls.

Demir et al. screened consecutive patients with T2DM for antibodies to HBV core and surface proteins (anti-HBc and anti-HBs). Of 545 eligible patients, 107 were anti-HBc and/or anti-HBs positive; 100 patients were randomly selected for inclusion in the study. The control group comprised 100 blood donors randomly selected from the 129 individuals (out of 642) who tested positive for anti-HBc and/or antiHBs. HBV DNA was found in the serum of 11 patients with T2DM, but in only 3 controls $(P<0.005)$. There were no significant betweengroup differences in age, sex, alcohol consumption, or history of surgery, transfusion, or HBV vaccination. Elevated serum alanine aminotransferase levels were found in 16 patients with T2DM versus 6 controls $(P<0.005)$, but there was no relationship between liver enzyme levels and $\mathrm{HBV}$ infection.

The authors suggest that the prevalence of HBV infection could partly explain the increased incidence of hepatocellular carcinoma in patients with T2DM.

Original article Demir M et al. (2008) The prevalence of occult hepatitis B virus infection in type 2 diabetes mellitus patients. Eur J Gastroenterol Hepatol 20: 668-673 